\title{
LITERATUR
}

\section{Europa - einig auf dem Weg ins All?}

\author{
Markus Hesse*
}

Die Europäische Union wird in der Europäischen Sicherheitsstrategie von Javier Solana als globaler Akteur beschrieben, der vor allem durch zivile Konfliktprävention eine neue multilaterale Weltordnung mitgestalten will. ${ }^{1}$ Um dieses Ziel zu erreichen und eine Hauptrolle in internationalen Ordnungsfragen spielen zu können, muss die Europäische Union über eigenständige Handlungsfähigkeiten verfügen. Unerlässlich sind dazu autonome Fähigkeiten der Aufklärung, Navigation und Kommunikation sowie Instrumente zur Führung ziviler und militärischer Einsatzkräfte. Beim Erwerb und Ausbau dieser Fähigkeiten spielt die Weltraumpolitik zunehmend eine Schlüsselrolle.

So erfuhren Raumfahrttechnologien in der Vergangenheit einen enormen Bedeutungszuwachs und werden heute in vielen verschiedenen Politik- und Wirtschaftsbereichen eingesetzt: in der Sicherheits- und Verteidigungspolitik ebenso wie in den Bereichen Verkehrsinfrastruktur, Umweltschutz und Datentransfer. Dabei ist die Struktur, die auf europäischer Ebene die rechtlichen, politischen und technischen Rahmenbedingungen systematisiert, sehr komplex. Ein zentraler Akteur ist die Europäische Weltraumorganisation (ESA), zu deren Aufgaben die Koordination und Durchführung nationaler und europäischer ziviler Weltraumprojekte zählen. Die Europäische Gemeinschaft besitzt hingegen Kompetenzen in Wirtschaft, Wissenschaft und Technik; in Ergänzung bemüht sich die Europäische Union - mit wechselndem Erfolg - um eine Gemeinsame Außen- und Si-
Stephan Hobe/Katharina Kunzmann/Thomas Reuter/Julia Neumann (Hrsg.): Rechtliche Rahmenbedingungen einer zukünftigen kohärenten Struktur der europäischen Raumfahrt, [Kölner Schriften zum Internationalen und Europäischen Recht, Band 13], LIT Verlag: Münster 2006, ISBN 3-8258-8963-7; 656 Seiten, 64,90 €.

Heiko Borchert (Hrsg.): Europas Zukunft zwischen Himmel und Erde. Weltraumpolitik für Sicherheit, Stabilität und Prosperität, [Schriftenreihe Vernetzte Sicherheit, Band 4], Nomos Verlagsgesellschaft: Baden-Baden 2005, ISBN 38329-1410-2; 170 Seiten, 24,90€.

cherheitspolitik (GASP) ihrer Mitgliedstaaten. Die Tätigkeitsfelder und Interessen der beiden Organisationen ESA und EG/EU überschneiden sich folglich zwangsweise in der Weltraumpolitik. Diesen Überschneidungen wurde bereits in mehreren wegweisenden $\mathrm{Ab}-$ kommen Rechnung getragen, wie dem EUESA Rahmenabkommen vom Mai 2004 und der Entschließung zur Europäischen Weltraumpolitik, die im Mai 2007 vom Weltraumrat, der gemeinsamen Tagung des Rates der Europäischen Union und des Rates der Europäischen Weltraumorganisation, gebilligt wurde. In diesen Dokumenten wird die immer enger werdende Zusammenarbeit der für die Weltraumpolitik relevanten Akteure hervorgehoben, um benutzerorientierte Großprojekte, wie das Navigationssystem ,Galileo“ oder das Erdbeobachtungsprogramm, Global Monitoring for Environment and Security" (GMES), gemeinsam erfolgreich umsetzen zu können.

\footnotetext{
* $\quad$ Markus Hesse, M.A., M.E.S., Wissenschaftlicher Mitarbeiter, Professur Europäische Integration, Technische Universität Chemnitz.

1 Javier Solana: Ein sicheres Europa in einer besseren Welt. Europäische Sicherheitsstrategie, Brüssel 2003, abrufbar unter: http://ue.eu.int/uedocs/cmsUpload/031208ESSIIDE.pdf (letzter Zugriff: 22.11.2007).
} 
Zwei kürzlich erschienene Bücher untersuchen die komplizierten Strukturen, die Akteure und die politischen Prozesse der europäischen Weltraumpolitik.

\section{Modelle für eine europäische Raumfahrt- struktur}

Mit den rechtlichen Rahmenbedingungen der europäischen Raumfahrt beschäftigt sich eine von Stephan Hobe, Katharina Kunzmann, Thomas Reuter und Julia Neumann herausgegebene Studie, die von Juni 2003 bis Mai 2005 am Institut für Luft- und Weltraumrecht der Universität Köln durchgeführt wurde. Erklärtes Ziel der Wissenschaftler war es, ,,in einem sich wandelnden organisatorischen Umfeld die rechtlichen Perspektiven der europäischen Weltraumadministration und damit das zukünftige Verhältnis der Europäischen Weltraumorganisation zur Europäischen Union zu evaluieren, sowie Vorschläge für mögliche künftige Strukturen zu erarbeiten“(Vorwort). Im Mittelpunkt der Untersuchung stehen die Akteure EG/EU und ESA, wobei mögliche Modelle einer Zusammenarbeit der beiden Organisationen vor einem rechtlichen und institutionellen Hintergrund geprüft werden. Die Studie ist in drei Abschnitte gegliedert: Im ersten Teil werden die Grundlagen der beiden Organisationen und ihre bisherige Zusammenarbeit vorgestellt und erläutert. Der zweite $\mathrm{Ab}$ schnitt stellt aktuelle Problemfelder in der Kooperation der beiden Organisationen dar und präsentiert Modelle für eine zukünftige Zusammenarbeit. Im dritten Teil werden Industrie- und Wirtschaftspolitik von EG/EU und ESA untersucht und ebenfalls die denkbaren Kooperationsmodelle geprüft.

Die Autoren beleuchten alle bedeutenden Problemstellungen, die sich auch aus einem verstärkten Engagement der Europäischen Union auf dem Gebiet der Raumfahrt ergeben haben. Hier sind zunächst die unterschiedlichen Mitgliedstaaten der beiden Organisationen zu nennen. Während die Europäische Union bereits auf 27 Mitgliedstaaten angewachsen ist, sind mit den EU-15, Norwegen und der
Schweiz derzeit 17 Staaten ESA-Mitglieder. Zudem unterscheiden sich die Entscheidungsprozesse in der ESA als einer, rein' internationalen Organisation stark von denen in der EG/EU. Darüber hinaus gibt es schließlich verschiedene Finanzierungsarten und unterschiedliche Auffassungen über die zivile und/ oder militärische Nutzung des Weltraums. Wie kann also in Anbetracht dieser Ausgangslage eine praktische Aufgabenabgrenzung zwischen den Organisationen auf der Basis des geltenden Rechts aussehen? Wie könnten geeignete zukünftige Strukturen aufgebaut sein?

Zur Beantwortung dieser und weiterer Fragen stellen die Autoren drei institutionelle Modelle sowie deren Rechtsgrundlagen und jeweiligen Vor- und Nachteile in der praktischen Umsetzung vor. Ein besonderes Verdienst der Studie ist sicherlich das umfassend und schlüssig begründete Ergebnis. Demnach ist das Beitrittsmodell, also ein Beitritt der EG/EU zur ESA, rechtlich und politisch kaum umzusetzen. Als kurzfristige Lösung präsentiert das Autorenteam das Kooperationsmodell unter Berücksichtigung der aktuellen Rechtsgrundlage, das eine verstärkte Zusammenarbeit der beiden Organisationen EG/EU und ESA vorsieht. Zu diesem Zweck empfehlen sie ein weiteres völkerrechtliches Abkommen zu unterzeichnen, das die Entscheidungsfindungsstruktur und die Durchführung von Raumfahrtprogrammen eindeutiger regelt, die einheitliche Interessenvertretung europäischer Raumfahrt gegenüber Drittstaaten klärt und sogar die Einbeziehung militärischer Raumfahrtprogramme ermöglichen soll. Als langfristige Lösung wird schließlich das Integrationsmodell präsentiert, das die Integration der ESA in die EG/EU und damit die primärrechtliche Errichtung einer EU-Raumfahrtagentur vorsieht. In der Studie wird mehrfach eine engere Kooperation von EG/EU und ESA angeregt, die eine unnötige Verdoppelung der Entscheidungsfindungsmechanismen vermeiden soll. 
Die Autoren berücksichtigen auch die Rechtsgrundlagen des EU-Verfassungsvertrages. Die Analysen sind jedoch nach wie vor relevant, da der Vertrag von Lissabon im Bereich der Raumfahrt durch den neuen Artikel 189 im Vertrag über die Arbeitsweise der Europäischen Union ähnliche rechtliche Rahmenbedingungen schaffen soll, wie sie im EUVerfassungsvertrag in Artikel III-254 vorgesehen waren. Insgesamt ermöglicht die Studie des Kölner Instituts Studienanfängern durch den Grundlagenteil einen Einstieg in Weltraumrecht und -politik und liefert Experten ein bedeutendes Werk zur aktuellen rechtlichinstitutionellen Debatte. Ein umfangreiches Literatur- und Dokumentenverzeichnis rundet die Studie ab, die in diesem Themenkomplex sicherlich als ein Standardwerk zu bezeichnen ist.

\section{Rolle des Weltraums in der vernetzten Sicher- heit}

Die von Heiko Borchert und Ralph Thiele herausgegebene Schriftenreihe, Vernetzte Sicherheit', in der bereits sieben Bände veröffentlicht worden sind, behandelt verschiedene aktuelle Themenbereiche der Sicherheitspolitik. Dabei liegt den Ausführungen stets der Ansatz einer vernetzten Sicherheit zugrunde. Gemeint ist damit die enge Kooperation aller Akteure im Bereich Sicherheit, deren Notwendigkeit sich aus den Beschleunigern der sicherheitspolitischen Vernetzung begründet: Neues Risiko- und verändertes Operationsbild, welche militärische und polizeiliche Aufgaben vermengen, und fortschreitende europäische Integration in Form von Erweiterung und Vertiefung. ${ }^{2}$ Die Realisierung vernetzter Sicherheitspolitik kann dabei auch eine europäische Weltraumpolitik einschlieBen.

Die Diskussion über eine zukünftige europäische Weltraum- und Sicherheitspolitik ist Ausgangspunkt der Beiträge des Sammel- bands. Dem Ansatz der vernetzten Sicherheit wird dadurch Rechnung getragen, dass der Themenkomplex ,Weltraum ' aus politischer, rechtlicher, militärischer und industrieller Sichtweise betrachtet wird. Der Herausgeber hat ein Autorenteam gewonnen, das zusätzlich zur ausgewiesenen Expertise eine gelungene Mischung aus Theorie und Praxis sowie verschiedener Fachbereiche aufweist. Dieser interdisziplinäre Ansatz in Verbindung mit dem gegebenen Praxisbezug stellt den aktuellen Stand der sicherheitspolitischen Forschung umfassend dar und ist somit eine hervorragende Weiterführung der zahlreichen theoriebasierten Debatten über den erweiterten Sicherheitsbegriff.

Unter diesen Gesichtspunkten erkennt Borchert die zunehmende sicherheitspolitische Bedeutung des Weltraums: ,Indem weltraumbasierte Fähigkeiten die globale Präsenz mit der jederzeitigen Möglichkeit zur lokalen Einwirkung verbinden, die Beschaffung und die Weitergabe von Informationen erleichtern sowie das Handlungsspektrum durch einen zusätzlichen Operationsraum erweitern, erfüllen sie in idealtypischer Weise die transformatorischen Kernanliegen der Entscheidungsüberlegenheit, der Vernetzung, der Beschleunigung der Entscheidungsprozesse und der gezielten Wirkung" (S. 11). Ein verstärktes staatliches Engagement bei der Erschließung des Weltraums führt nach Borchert $\mathrm{zu}$ einem erweiterten nationalen Handlungsspielraum. Zusätzlich seien die positiven Auswirkungen von Investitionen in Technologien, wie beispielsweise der Informations- und Kommunikationstechnologie, beim Aufbau weltraumbasierter Fähigkeiten zu berücksichtigen.

Der vorliegende Sammelband folgt diesen Bezugspunkten, indem die ersten Beiträge nationale und internationale Weltraum-Außenpolitiken und deren Verbindungen zur

2 Heiko Borchert: Vernetzte Sicherheitspolitik und die Transformation des Sicherheitssektors: Weshalb neue Sicherheitsrisiken ein verändertes Sicherheitsmanagement erfordern, in: Vernetzte Sicherheit. Leitidee der Sicherheitspolitik im 21. Jahrhundert, Schriftenreihe Vernetzte Sicherheit, Band 1, Hamburg 2004, S. 53-79. 
Sicherheitspolitik beleuchten. Untersucht werden in diesem Zusammenhang Kooperationsbeziehungen innerhalb der Europäischen Union (Thomas Jäger/Mischa Hansel) sowie die Zusammenarbeit zwischen Europa, den USA, Russland und China (Niklas Reinke). Weitere Schwerpunkte bilden Industriestrategie und -politik (Achim Bachem/Karsten Beneke/Helmut Süß/Thomas Dittler/Dieter Hayn/Joachim Klein), weltraumrelevante industrielle Kompetenzen (Robert Haberberger/Gerd Hofschuster) und die Rolle von $\mathrm{Pu}-$ blic-Private-Partnerships (Martin U. Ripple). Weitere Beiträge zu rechtlichen (Kai-Uwe Schrogl) und militärischen Fragestellungen (Ralph Thiele) sowie zu kommerziellen weltraumgestützten Dienstleistungen (Reinhard H. Czichy/Heiko Borchert) runden den Band $\mathrm{ab}$.

Auffällig ist bei einigen Abhandlungen, dass kritische Meinungen, zum Beispiel zu einer Militarisierung des Weltraums oder einem neuen Wettrüsten im All, kaum berücksichtigt werden. Die Autoren untermauern ihre Thesen mit stichhaltigen und gleichzeitig nachvollziehbaren Argumenten, ohne jedoch jeweils explizit die Gegenmeinungen, die als Gegensatz beispielsweise in Friedensforschung und Sicherheitspolitik existieren, aufzunehmen. Die sicherheitspolitische Nutzung des Weltraums wird größtenteils vorausgesetzt und könnte an einigen Stellen stärker hinterfragt werden.

Herausragende Merkmale des Bandes sind sicherlich seine Praxisrelevanz und seine Vielseitigkeit. Die Entwicklungen in der Weltraumpolitik und -technologie schreiten schnell voran. Es ist daher zu hoffen, dass die Herausgeber und Autoren sich in Zukunft weiterhin dem Weltraum widmen werden.

\section{Zukunft europäischer Weltraumpolitik}

Die Weltraumpolitik in Europa befindet sich momentan in einer interessanten und spannenden Phase, da Finanzierung und GovernanceStruktur der beiden Flaggschiffprojekte, des Satellitennavigationsprogramms ,Galileo“ und des Erdbeobachtungsprogramms, GMES', von den beteiligten Akteuren im kommenden Jahr konkretisiert werden sollten, um eine erfolgreiche Umsetzung zu ermöglichen. Erfolg oder Misserfolg in der Zusammenarbeit zwischen EG/EU und ESA werden eine entscheidende Rolle für die zukünftige Entwicklung und die Konkurrenzfähigkeit gegenüber anderen Weltraumnationen, vor allem USA und Russland, aber auch China, Indien und Japan, spielen.

Doch wie kann und wird eine kohärente rechtliche und politische Struktur der europäischen Raumfahrt aussehen? Eine konkrete Kooperation findet zum Beispiel beim Projekt, Galileo derzeit mit wechselndem Erfolg statt. Nach Aussage des Europäischen Wirtschafts- und Sozialausschusses hätte jedoch ,eine Aufgabe des Galileo-Projekts verheerende Folgen für die Europäische Union". 3 Auf der Sitzung am 21. und 22. Juni 2007 bekräftigte auch der Europäische Rat nochmals die Bedeutung von ,Galileo“ als ein Schlüsselprojekt der Europäischen Union. Der Rat, Verkehr, Telekommunikation und Energie" hat die Europäische Kommission unter deutscher Ratspräsidentschaft aufgefordert, neue Möglichkeiten zur Finanzierung zu erarbeiten. In einer Mitteilung der Europäischen Kommission wurden daraufhin neue politische, finanzielle und verwaltungstechnische Vorschläge präsentiert, die eine Weiterführung des Projekts sichern sollen. ${ }^{4}$ Die Kommission geht in ihren aktuellen Berechnungen von Beschaffungskosten in Höhe von 3,4 Mrd. Euro und einer vollständigen Betriebsfähigkeit des Systems im Jahr

3 Europäischer Wirtschafts- und Sozialausschuss: Stellungnahme zu der „Mitteilung der Kommission an den Rat, das Europäische Parlament, den Europäischen Wirtschafts- und Sozialausschuss und den Ausschuss der Regionen - GALILEO am Scheideweg: die Umsetzung der europäischen GNSS-Programme“, KOM(2007) 261 endgültig, 16.05.2007, in: Amtsblatt der EU, Nr. C 256 vom 27.10.2007, S. 73.

4 Europäische Kommission: Mitteilung der Kommission an das Europäische Parlament und den Rat: Galileo: Die europäischen GNSS-Programme mit neuem Profil, KOM(2007) 534 endgültig, 19.09.2007. 
2013 aus, wobei sie auch potenzielle Risiken, wie Konzeptions-, Errichtungs- und Marktrisiko, berücksichtigt. Ende November/Anfang Dezember 2007 kam es schließlich im Verkehrsrat zu einer Einigung über, Galileos ' $\mathrm{Zu}$ kunft und die Regeln zur Auftragsvergabe. Mehrere EU-Organe und -Institutionen sind also maßgeblich in aktuelle und künftige Entwicklungen einer europäischen Raumfahrtpolitik eingebunden.

Fortschritte beim Ausbau einer kohärenten politischen und rechtlichen Struktur hängen in diesem Politikbereich aber auch stark von nationalen Interessen ab. Tendenziell haben Staaten mit umfangreichem Weltraumprogramm und umsatzstarker Raumfahrtindustrie, wie Frankreich und Deutschland, auch ein größeres Interesse an neuen Entwicklungen. Weitere Impulse könnte vor allem die französische EU-Ratspräsidentschaft im zweiten Halbjahr 2008 bringen, die Weltraumpolitik und -projekte auf die Agenda der Europäischen Union setzen wird.

\title{
Europäische Nachbarschaftspolitik und externe Demokratieförderung durch die EU im Spiegel der Forschung
}

\author{
Anne Faber*
}

Die Europäische Nachbarschaftspolitik (ENP) ist seit den ersten Ausgangsüberlegungen zu einer systematischeren und kohärenteren Politik der Europäischen Union gegenüber ihren neuen Nachbarstaaten in Ost- und Südeuropa in der zweiten Hälfte der 1990er Jahre zunehmend in den Vordergrund politischer und wissenschaftlicher Debatten über die europäische Außen- und Erweiterungspolitik gerückt. Es stellt sich jedoch die Frage, ob dies der einzig gangbare Weg bei der schwierigen Konsenssuche zwischen der ,Erweiterungsmüdigkeit beziehungsweise der vielfach zitierten, scheinbar erschöpften ,Absorptionskapazität ‘ der Europäischen Union einerseits und dem erklärten Wunsch nach einer baldigen Vollmitgliedschaft seitens einer Reihe von Staaten in Mittel- und Osteuropa, aber auch in Südeuropa andererseits, ist. Gleichzeitig werden bei der konkreten Umsetzung und Anwendung der ENP die Brüche und Grenzen dieser Politik deutlich, was eine Reihe von Fragen nicht nur zu ihrer Konzeption und ihrem aktuellen Stand, sondern auch ihrer zukünftigen Ent-
Johannes Varwick und Kai-Olaf Lang (Hrsg.): European Neighbourhood Policy. Challenges for the EU-Policy Towards the New Neighbours, Barbara Budrich Publishers: Opladen \& Farmington Hills 2007, ISBN 978-3-86649-125-0; 229 Seiten, $24,90 €$.

Annette Jünemann und Michèle Knodt (Hrsg.): Externe Demokratieförderung durch die Europäische Union. European External Democracy Promotion, [Schriftenreihe des Arbeitskreises Europäische Integration e.V., Band 58], Nomos Verlagsgesellschaft: Baden-Baden 2007, ISBN 978-3-8329-2794-3; 376 Seiten, 49,00€.

Martin Große Hüttmann, Matthias Chardon und Siegfried Frech (Hrsg.): Das neue Europa, WOCHENSCHAU Verlag: Schwalbach/Ts. 2007, ISBN 978-3-89974355-5; 298 Seiten, 16,80 €.

wicklung aufwirft. Aber auch das außenpolitische Handeln der Europäischen Union insgesamt steht auf dem Prüfstand: Wie steht es um die Umsetzung des erklärten Ziels, De-

* Dr. Anne Faber, ehem. Wissenschaftliche Mitarbeiterin, Jean Monnet Lehrstuhl Prof. Dr. Wolfgang Wessels, Forschungsinstitut für Politische Wissenschaft und Europäische Fragen, Universität zu Köln. 